Research Article

\title{
Seepage Damage Statistical Model of Filled Fractured Rock considering Structural Surface and Failure Characteristic
}

\author{
Xinyu Liu ${ }^{(D)}{ }^{1}$ Yuan Tian $\left(\mathbb{D},{ }^{2,3}\right.$ and Zhende $\mathrm{Zhu}^{2,3}$ \\ ${ }^{1}$ College of Civil Engineering and Architecture, Zhejiang University of Water Resources and Electric Power, \\ Hangzhou 310018, China \\ ${ }^{2}$ Key Laboratory of Ministry of Education for Geomechanics and Embankment Engineering, Hohai University, \\ Nanjing 210098, China \\ ${ }^{3}$ Jiangsu Research Center for Geotechnical Engineering, Hohai University, Nanjing 210098, China
}

Correspondence should be addressed to Xinyu Liu; liuxy267@zjweu.edu.cn

Received 15 September 2021; Accepted 5 November 2021; Published 22 November 2021

Academic Editor: Jie Liu

Copyright (c) 2021 Xinyu Liu et al. This is an open access article distributed under the Creative Commons Attribution License, which permits unrestricted use, distribution, and reproduction in any medium, provided the original work is properly cited.

Damage theory studies the whole process of initiation, propagation, and instability of microcracks in materials and provides an important basis for the estimation of the risk of materials. Therefore, it is assumed that the rock microunit strength is the damage variable of the medium and obeys the Weibull distribution. According to the tensile failure characteristics of filled fractured rock under the action of seepage stress, the maximum tensile strain criterion is used to define the rock microunit strength parameters, and the equivalent elastic modulus of the fractured rock is used to establish a new damage statistical model. This paper mainly studies the rationality and feasibility of using this new constitutive model to describe the seepage failure process and damage characteristics of filled fractured rock. The results indicate that (1) the accuracy of the equivalent elastic modulus is affected by the confining pressure and the characteristics of the structural surface. In the elastic phase, using the equivalent elastic modulus, $E_{V R H}$ has better fit. In the plastic phase, it is better to use the $E_{V}$ parameter. (2) The established Weibull distribution statistical model can better calculate the stress-strain curve of fractured rocks with weak and soluble fillings. (3) The rock strength characteristics affected by different stress conditions and different filling fracture states calculated by the model are the same as the experimental data. (4) The model using equivalent elastic modulus parameters reflects the threshold characteristics of rock failure and the damage evolution process. After comparison, it is found that the model can accurately calculate the final damage value of the fractured rock with weak and soluble filling. However, the final damage value used to calculate the fractured rock of the hydraulic material filling is much higher and inaccurate.

\section{Introduction}

In geotechnical engineering, a key theoretical problem to solve the stability prediction and evaluation of surrounding rock is the rock constitutive model. Since Dougill [1] first applied damage mechanics to the field of rock mechanics in 1976, scholars have established various models based on damage mechanics theory [2-5]. Literature [6-12] opened up new ideas in the study of rock constitutive models. According to the randomness of the distribution of the cracks in the rock, it is proposed that the damage variable of the medium obeys the Weibull distribution, and the continuous damage theory and the statistical strength theory are used to solve the problem. This statistical damage model can better reflect the rock deformation than similar models established by predecessors.

Through the establishment of the Weibull distribution damage statistical constitutive model, Wang et al. [13] realized the fitting calculation of the residual strength of the rock. Based on the comparison between the damage constitutive model of the power function distribution and the damage constitutive model of the Weibull distribution, Chen et al. [14] found that the characteristics of rock microunit strength can be described more reasonably by the Weibull distribution statistical model. Using the three-parameter Weibull distribution model, Zuo et al. [15] realized 
the failure strength calculation of double-cut rock under thermomechanical loading. Based on the Weibull distribution model, $\mathrm{Li}$ et al. [16] realized the fitting of the progressive failure of the rock, and Sun and Zhu [17] studied the relationship between the stress-strain of the rock and the wave velocity. Xu et al. [18] assumed that the heterogeneity of the intact rock block obeys the Weibull distribution and performed a numerical analysis on the compressive strength of prefabricated jointed rocks. Many scholars [19-27] have realized that rock failure and its penetration are closely related to micro damage evolution and macro crack generation.

From the study of the seepage characteristics of rock mass joints by many scholars, it can be known that the existence of fillings makes the seepage characteristics of fractured rocks significantly different from those of unfilled fractured rocks. Under the action of hydrodynamic force, the filling material in the fracture is affected by slight or severe changes in the rock internal structure. This leads to dissolution and migration of the filling material, which ultimately affects the strength, failure, and permeability characteristics of the fractured rock with fillings. The purpose of this study is to investigate the strength and damage characteristics of fractured sandstone with fillings under the action of seepage stress, establish a reasonable and feasible constitutive model, and verify the reliability of the model by analyzing the rock damage softening characteristics.

In the indoor seepage test, hydrodynamic pressure flows into the filled fractures in the rock. This part of the water pressure forms a certain pore wall pressure and eventually leads to tensile cracks in the rock. The tensile failure occurs in the rock filled with fractures [28]. Therefore, according to the tensile failure characteristics of the cracked rock, based on the maximum tensile strain criterion, the rock microunit strength is the damage variable, and the equivalent elastic modulus parameter is used. In this paper, a new Weibull distribution statistical damage model is established, which is used to characterize the seepage damage and failure process and characteristics of fractured rock with fillings under hydrodynamic force.

\section{Seepage Damage Statistical Model}

2.1. Model Parameter. Voigt (1910) proposed an average model, assuming that the strain of various minerals caused by additional stress in the rock is uniform. Figure 1(a) describes such an idealized strain distribution. As shown in the figure, the distribution direction of various minerals arranged in parallel in the rock is consistent with the direction of force. The rock contains many kinds of minerals, and $i$ represents one of the minerals. Its rock volume percentage is $V_{i}(i=1, \ldots$, $N$ ), its bulk modulus is $K_{i}$, and its shear modulus is $\mu_{i}$. According to the space volume average method of the Voigt model, the equivalent bulk modulus $K_{V}$ of the rock can be obtained. Another average model was proposed by Reuss (1929). In his model, various minerals in the rock are subjected to the same stress, but their strain fields are not uniform. Figure 1(b) depicts such an idealized stress distribution. As shown in the figure, the various minerals in the rock are arranged in layers, but the layering direction is perpendicular to the stress direction. The calculated bulk modulus at this time is expressed in $K_{R}$. After proof, Hill (1952) proposed that the upper limit of the equivalent elastic parameter estimated value is the result of the Voigt model, and the result obtained by the Reuss model is the lower limit of the estimated value of the parameter. The parameters obtained by actual rock measurement must fall between these two estimated values [29]. Take the arithmetic average of the results of these two models, and the resulting bulk modulus is represented by $K_{V R H}$.

According to (1) and (2), the bulk modulus $K_{V}$ of the Voigt model and the bulk modulus $K_{R}$ of the Reuss model can be calculated, respectively.

$$
\begin{aligned}
K_{V} & =\sum_{i=1}^{N} K_{i} \cdot V_{i}, \\
K_{R}^{-1} & =\sum_{i=1}^{N} K_{i}^{-1} \cdot V_{i},
\end{aligned}
$$

and the multiphase equivalent rock bulk modulus $K_{V R H}$ is

$$
K_{V R H}=\frac{1}{2}\left(K_{R}+K_{V}\right) .
$$

According to the equation between bulk modulus and elastic modulus,

$$
E=3 K(1-2 v) .
$$

The equivalent elastic modulus $E_{V}$ and $E_{V R H}$ can be calculated.

2.2. Constitutive Model. According to the phenomenological method of continuum mechanics, based on the apparent phenomena of materials, and applying Lemaitre's strain equivalence principle, a mechanical analysis model of damage coupling can be established.

$$
\sigma^{\prime}=\frac{\sigma}{1-D}
$$

where $D$ is the damage variable. It reflects the damage degree and damage history of the medium.

Damage theory studies the whole process of materials from primary defects to macroscopic cracks and fractures, and the mesoscopic nonuniformity can be characterized by macroscopically measurable statistical distribution. $F$ is a certain macroscopic characteristic parameter, which is defined here as the rock microunit strength. Its statistical distribution follows the Weibull distribution function. It is assumed that the damage body is linearly elastic before the damage; the macro elastic modulus is $E$. Its probability density, damage parameter, and constitutive relation are written as

$$
\begin{aligned}
P(F) & =\frac{m}{F_{0}}\left(\frac{F}{F_{0}}\right)^{m-1} \exp \left[-\left(\frac{F}{F_{0}}\right)^{m}\right], \\
D & =1-\exp \left[-\left(\frac{F}{F_{0}}\right)^{m}\right],
\end{aligned}
$$




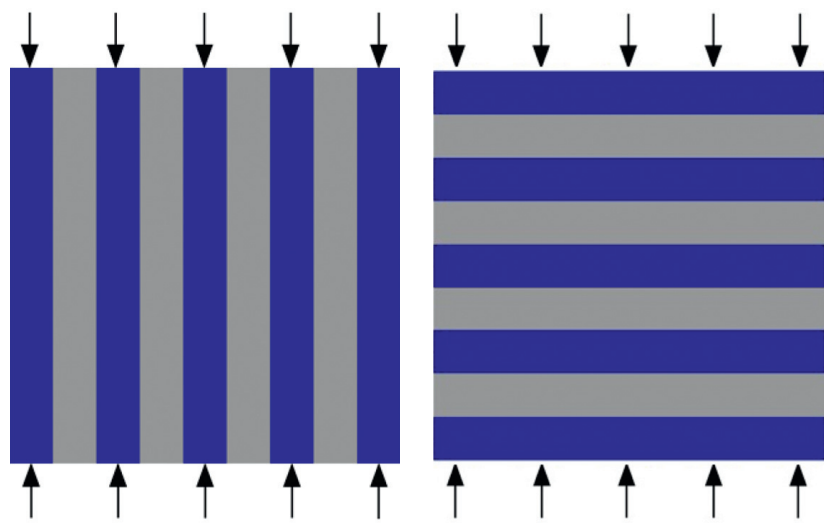

(a)

(b)

FIgure 1: (a) Voigt's equal strain model. (b) Reuss's equal strain model.

where $m$ and $F_{0}$ are the Weibull distribution parameters.

According to the results of the indoor seepage test, it is found that the fractured rock with filling is mainly tensile failure under the action of seepage stress, so the maximum tensile strain theory is used to establish $F$.

$$
F=f\left(\sigma^{\prime}\right)=\sigma_{1}^{\prime}-2 \mu\left(\sigma_{2}^{\prime}+\sigma_{3}^{\prime}\right) .
$$

According to the effective stress principle, Hooke's law, and equation (5), putting equation (4) into them, get

$$
\begin{aligned}
& F=E \varepsilon_{1}, \\
& \sigma_{1}=E \varepsilon_{1}(1-D)+2 \mu \sigma_{3}+(1-2 \mu) p .
\end{aligned}
$$

It is known that the rock softening damage stress-strain curve must have an extreme value, and the extreme value is at the peak point of the curve. At this time, the stress is $\sigma_{1}=\sigma_{1 \text { max }}$, the strain is $\varepsilon_{1}=\varepsilon_{1 \max }$, and the rock microunit strength is $F_{\max }$. By calculating the derivative $d \sigma_{1 \max } / d \varepsilon_{1 \max }$ of equation (9), the result is

$$
\begin{array}{r}
\frac{\mathrm{d} \sigma_{1 \max }}{\mathrm{d} \varepsilon_{1 \max }}=0, \\
1-\frac{m E \varepsilon_{1 \max } F_{\max }^{m-1}}{F_{0}^{m}}=0 .
\end{array}
$$

So, get

$$
\begin{aligned}
F_{0}^{m} & =m F_{\max }^{m}, \\
F_{0} & =E \varepsilon_{1 \max } \cdot m^{1 / m} .
\end{aligned}
$$

At the peak point, by substituting equation (12) into equation (9), the correlation relation becomes

$$
m=\frac{1}{\ln \left(E \varepsilon_{1 \max }\right)-\ln \left[\sigma_{1 \max }-2 \mu \sigma_{3}-(1-2 \mu) p\right]} .
$$

In the triaxial seepage experiment, the relation between axial pressure $\sigma_{1}$, confining pressure $\sigma_{3}$, and axial bias $\sigma_{1 t}$ is

$$
\sigma_{1 t}=\sigma_{1}-\sigma_{3} .
$$

The initial axial deformation $\varepsilon_{1 o}$ caused by the confining pressure before the axial pressure is

$$
\varepsilon_{1 o}=\frac{1-2 \mu}{E}\left(\sigma_{3}-p\right)
$$

The true axial strain $\varepsilon_{1}$ is

$$
\varepsilon_{1}=\varepsilon_{1 t}+\varepsilon_{1 o}
$$

where $\varepsilon_{1 t}$ is the measured test strain.

Substituting equations (16)-(18) into equations (9), (14), and (15), the new parameters calculation methods are

$$
\begin{aligned}
F & =\left[E \varepsilon_{1 t}+(1-2 \mu)\left(\sigma_{3}-p\right)\right] \\
F_{0} & =\left[E \varepsilon_{1 t \max }+(1-2 \mu)\left(\sigma_{3}-p\right)\right] \cdot m^{1 / m} \\
m & =\frac{1}{\ln \left[E \varepsilon_{1 t \max }+(1-2 \mu)\left(\sigma_{3}-p\right)\right]-\ln \left[\sigma_{1 t \max }+(1-2 \mu)\left(\sigma_{3}-p\right)\right]}
\end{aligned}
$$

Finally, the damage softening model constitutive is 


$$
\sigma_{1 t}=\left[E \varepsilon_{1 t}+(1-2 \mu)\left(\sigma_{3}-p\right)\right] \exp \left[-\left(\frac{1}{m}\right)\left(\frac{E \varepsilon_{1 t}+(1-2 \mu)\left(\sigma_{3}-p\right)}{E \varepsilon_{1 t \max }+(1-2 \mu)\left(\sigma_{3}-p\right)}\right)^{m}\right]+(2 \mu-1)\left(\sigma_{3}-p\right)
$$

\section{Fitting Calculation}

To verify the application conditions of different elastic modulus and the rationality of establishing the Weibull model, the experimental data in the literature are cited for fitting calculation work. According to the experiment in literature [28], the seepage experiment results of single fractured sandstone filled with gypsum mortar and single fractured sandstone filled cement mortar were obtained at different confining pressures of $10 \mathrm{MPa}$ and $20 \mathrm{MPa}$. The calculation results are as shown in Table 1 and Figures 2-9.

3.1. Stress-Strain Curve. In Figures 2-5, they are the damage model calculated curve and the experimental curve. The results show that

(1) In the elastic phase, the damage model uses $E_{V R H}$ parameters. After the elastic phase, the model adopts $E_{V R H}$ parameters. After comparison, if the $E_{V}$ parameter is used in the elastic section, the model calculation curve is much higher than the test curve value, and the fit is poor. It shows that in the elastic stage, the structural surface characteristics of the rock have no obvious influence on the strength. The elastic segment curve calculated by using $E_{V R H}$ parameters fits well with the test curve. At low confining pressure, the model calculation curve for filling fractured rock is slightly lower than the experimental curve. At high confining pressure, the model calculation curve and the test curve are in good agreement.

(2) The shape of the model curve is basically the same as that of the test curve. The calculated curve can reflect the strength changes characteristics of the fractured rock affected by different fillings; at the same time, the model curve also reflects the strength changes characteristics of the fractured rock with fillings under different confining pressures and the strength characteristics of the cracked rock under different structural surfaces.

(3) Starting from the plastic deformation stage, the model uses the $E_{V}$ equivalent modulus. Under the same stress, the model strain value is smaller than the test strain value. When drawing the model curve, it is found that the filled fractured rock elastic modulus is between the $E_{V}$ value and the $E_{V R H}$ value but is closer to the $E_{V}$ value. It shows that the characteristics of the structural plane have more influence on the rock strength. Secondly, another reason is that the test adopts the principle of transient pulse technology, which causes a certain creep of the sample. In the postpeak phase of the curve, since the established model uses damage factors to modify the loading process, the revised Weibull distribution model can better match the actual situation after the rock is broken.

3.2. Damage Characteristics. Comparing the rock damage evolution curve obtained from the experiment data with the ones obtained from the calculation model, the rock damage variable change rule with strain can be obtained, and the accuracy and correctness of the statistical damage model can be verified.

Therefore, transforming equation (9) can get

$$
\begin{aligned}
& D=\frac{\left[E \varepsilon_{1}-\sigma_{1}+2 \mu \sigma_{3}+(1-2 \mu) p\right]}{E \varepsilon_{1}}, \\
& D=\frac{\left(E \varepsilon_{1 t}-\sigma_{1 t}\right)}{\left[E \varepsilon_{1 t}+(1-2 \mu)\left(\sigma_{3}-p\right)\right]} .
\end{aligned}
$$

The $D-\varepsilon_{1 t}$ test data curves are obtained, as shown in Figures 6 and 7 .

Figures 6 and 7 are the damage variable evolution curves calculated according to the test data. In the low strain compaction stage, the curve shows a shape of increasing first and then decreasing. At this time, the microcracks in the filled fracture rock are closed under stress, that is, dilatancy hardening. After entering the stage of linear elasticity, the damage of the rock shows a monotonous increasing trend. The slope of the curve is basically consistent with the slope of the growth curve in the initial stage, indicating that the softening rate of the rock is consistent under the hydrodynamic force in the seepage test. At the same time, since the rock damage is the overall unstable failure caused by the fracture cone structure developed from the end of the single fracture, the damage value of the rock is not high when the failure is caused, and the material failed to achieve full damage.

According to the Weibull distribution model established in this paper, the theoretical damage value can be calculated as

$$
D=1-\exp \left[-\left(\frac{F}{F_{0}}\right)^{m}\right]
$$

The corresponding theoretical curves of $D-\varepsilon_{1 t}$ are shown in Figures 8 and 9.

In Figures 8 and 9, the model has considered the damage of the filled fracture part when calculating the elastic modulus parameters. At high confining pressure, when the cracks are filled with cement mortar, the rock damage in the elastic stage is lower than the original damage because of the hardening effect of the confining pressure and the hydraulic characteristics of the filling material. Therefore, there is basically no rock damage value in the elastic stage of the calculation model. However, at low confining pressure, the damage value of fractured rock filled with gypsum mortar is 
TABLE 1: The equivalent elastic parameters of the filled fractured rock.

\begin{tabular}{|c|c|c|c|c|c|}
\hline Samples & $K_{V}(\mathrm{GPa})$ & $K_{R}(\mathrm{GPa})$ & $E_{V}(\mathrm{GPa})$ & $E_{R}(\mathrm{GPa})$ & $E_{V R H}(\mathrm{GPa})$ \\
\hline $1-1$ & 4.27 & 1.01 & 6.41 & 1.82 & 4.11 \\
\hline $1-2$ & 4.44 & 1.83 & 7.20 & 3.30 & 5.25 \\
\hline $1-3$ & 4.10 & 0.58 & 6.15 & 1.04 & 3.59 \\
\hline $1-4$ & 4.27 & 1.15 & 6.92 & 2.08 & 4.50 \\
\hline $1-5$ & 3.92 & 0.40 & 4.71 & 0.72 & 2.71 \\
\hline $1-6$ & 4.09 & 0.84 & 6.62 & 1.51 & 4.06 \\
\hline $2-1$ & 4.27 & 0.60 & 6.15 & 1.08 & 3.62 \\
\hline $2-2$ & 4.44 & 0.87 & 7.20 & 1.57 & 4.38 \\
\hline $2-3$ & 4.09 & 0.32 & 6.14 & 0.58 & 3.36 \\
\hline $2-4$ & 4.27 & 0.47 & 7.67 & 0.85 & 4.26 \\
\hline $2-5$ & 3.92 & 0.22 & 6.11 & 0.39 & 3.25 \\
\hline $2-6$ & 4.08 & 0.33 & 6.85 & 0.59 & 3.72 \\
\hline
\end{tabular}

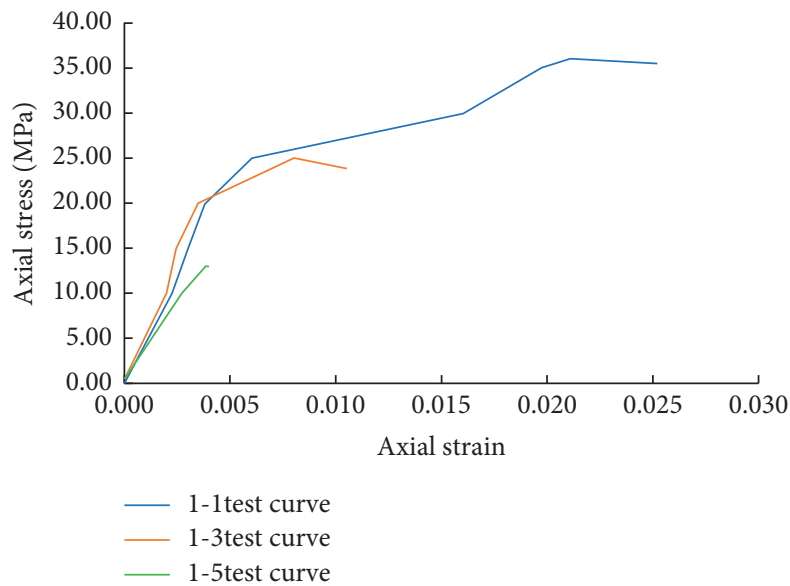

(a)

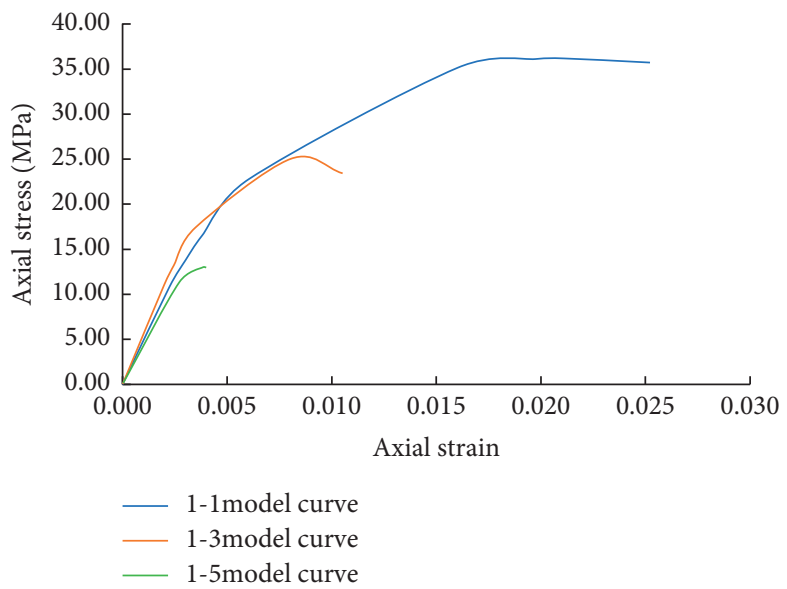

(b)

FIgURE 2: Test stress-strain curve((I) of filled cracked rocks with gypsum mortar $\left(\sigma_{3}=10 \mathrm{MPa}\right)$.

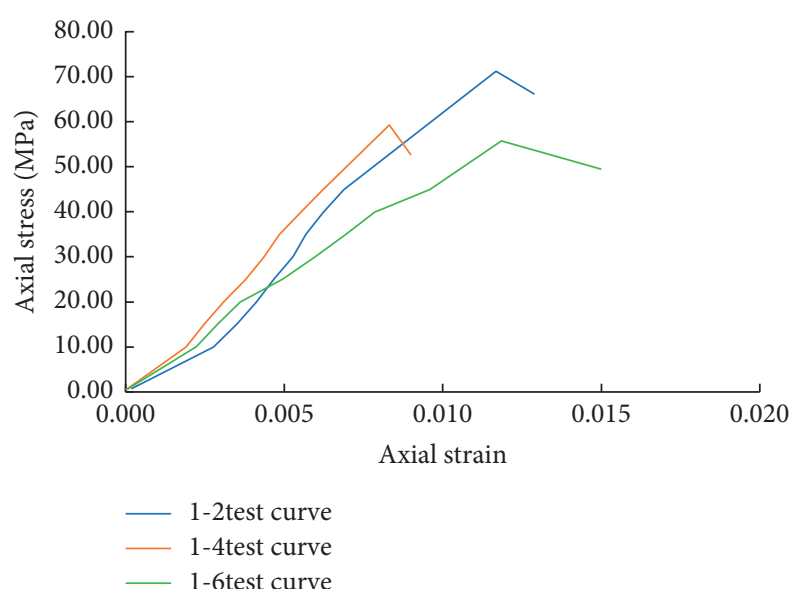

(a)

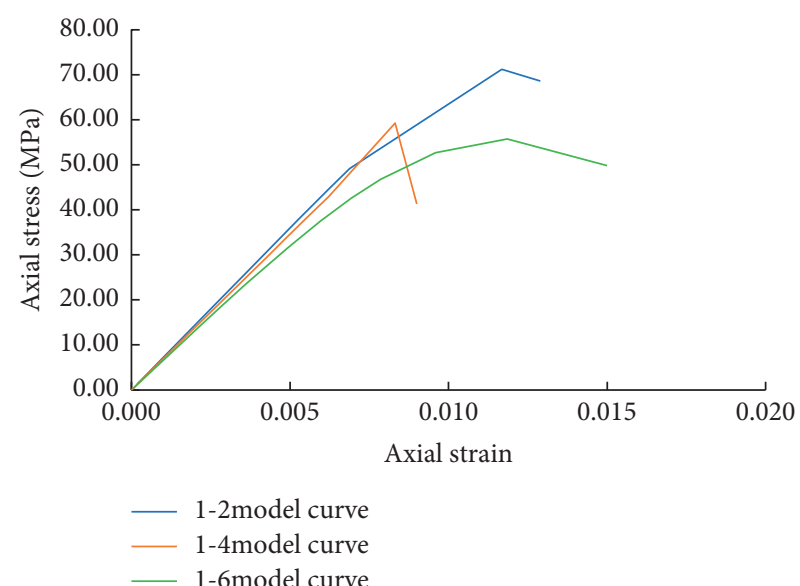

(b)

Figure 3: Stress-strain curve (II) of filled cracked rocks with gypsum mortar $\left(\sigma_{3}=20 \mathrm{MPa}\right)$.

greater than the original damage. Therefore, the calculated value starts to increase from the elastic stage.

In Figure 8, the damage theoretical curves of 1-1, 1-3, and $1-5$ rocks are mainly convex, while the ones of $1-2,1-4$, and 1-6 rocks are mainly concave. The growth rate of damage decreases slightly with the increase of confining pressure. As the length of the filling crack increases, the strain value of the cracked rock decreases during failure. In 


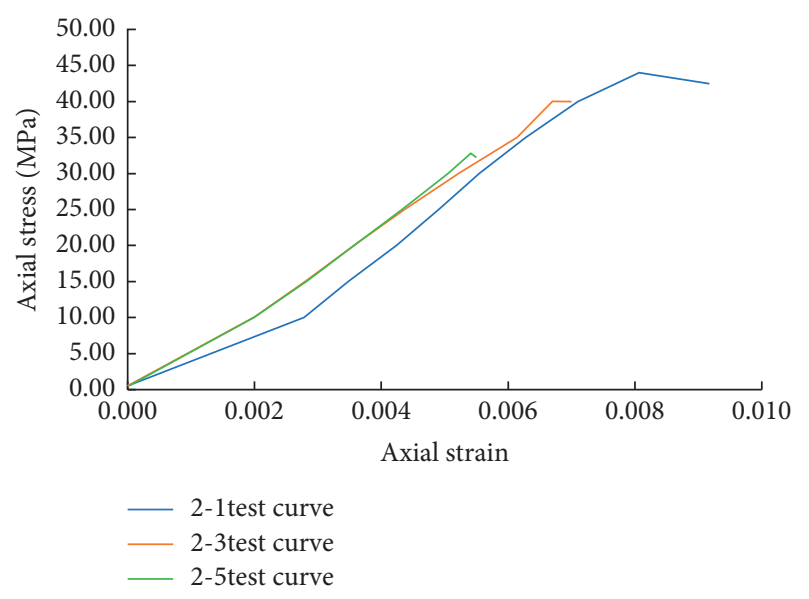

(a)

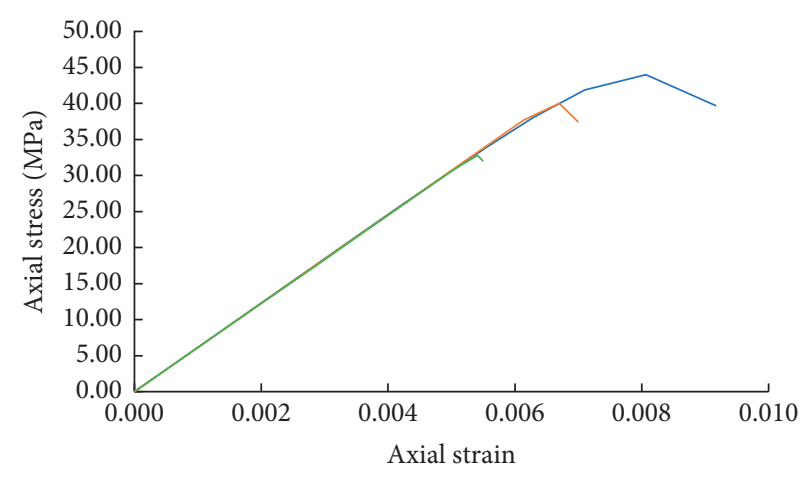

2-1model curve
2-3model curve
- 2-5model curve

(b)

FIGURE 4: Stress-strain curve (I) of filled cracked rocks with cement mortar $\left(\sigma_{3}=10 \mathrm{MPa}\right)$.

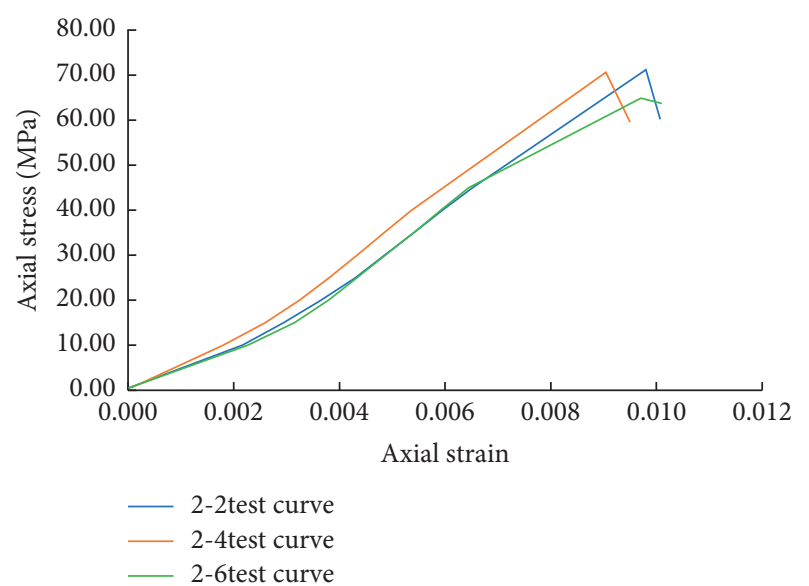

(a)

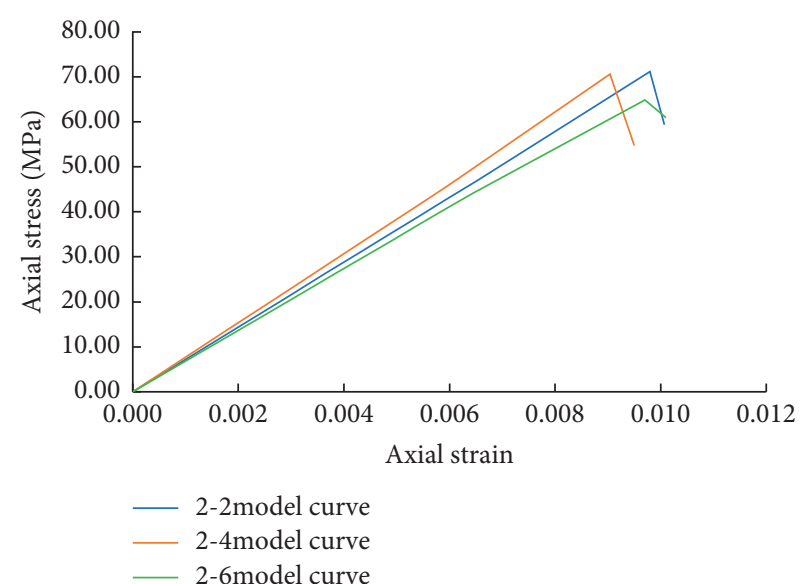

(b)

Figure 5: Stress-strain curve (II) of filled cracked rocks with cement mortar $\left(\sigma_{3}=20 \mathrm{MPa}\right)$.

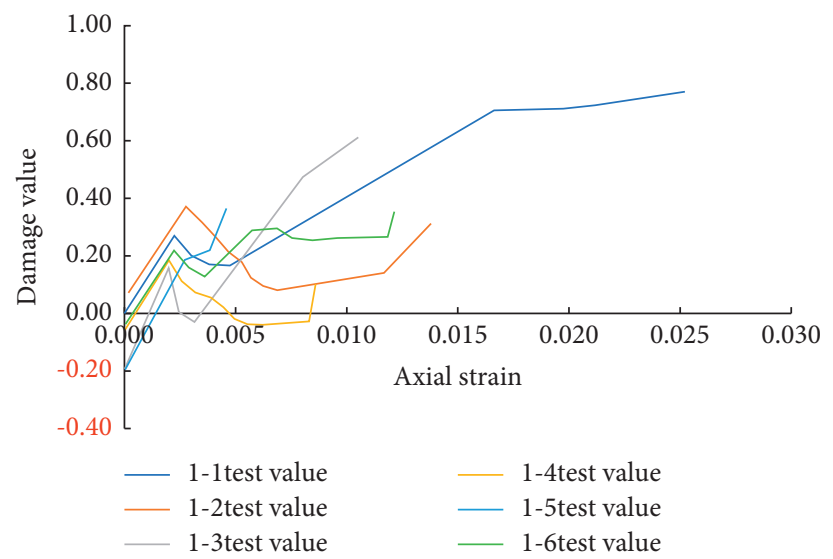

FIgURE 6: $D-\varepsilon_{1 t}$ test curve of filled cracked rocks with gypsum mortar.

Figure 9, the damage theoretical curve is zero in the compaction stage. From the beginning of plastic deformation, the damage begins to accumulate rapidly. When the initial damage occurs, with the increase of the confining pressure, the initial strain of the rock increases. It shows that the confining pressure hardening effect is significant. 


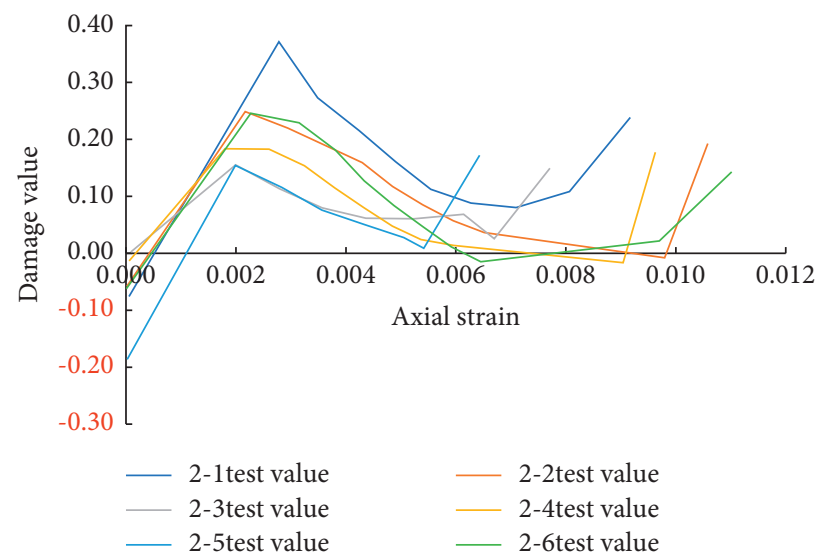

FIgURE 7: $D-\varepsilon_{1 t}$ test curve of filled cracked rocks with cement mortar.

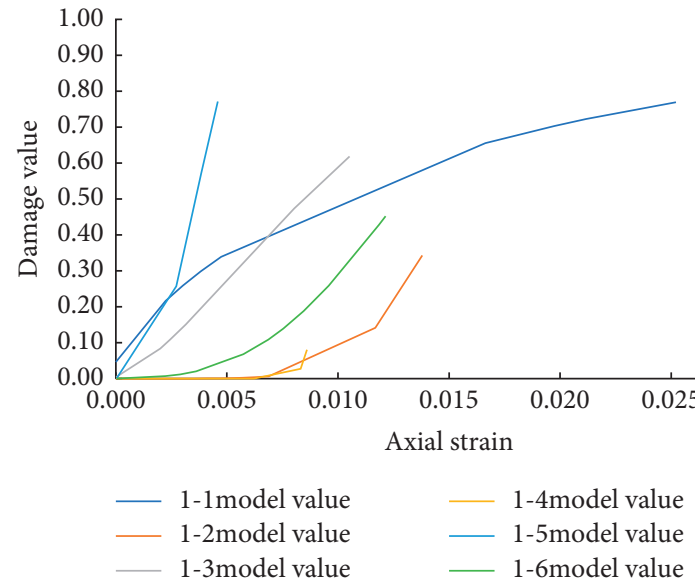

Figure 8: $D-\varepsilon_{1 t}$ theoretical curve of filled cracked rocks with gypsum mortar.

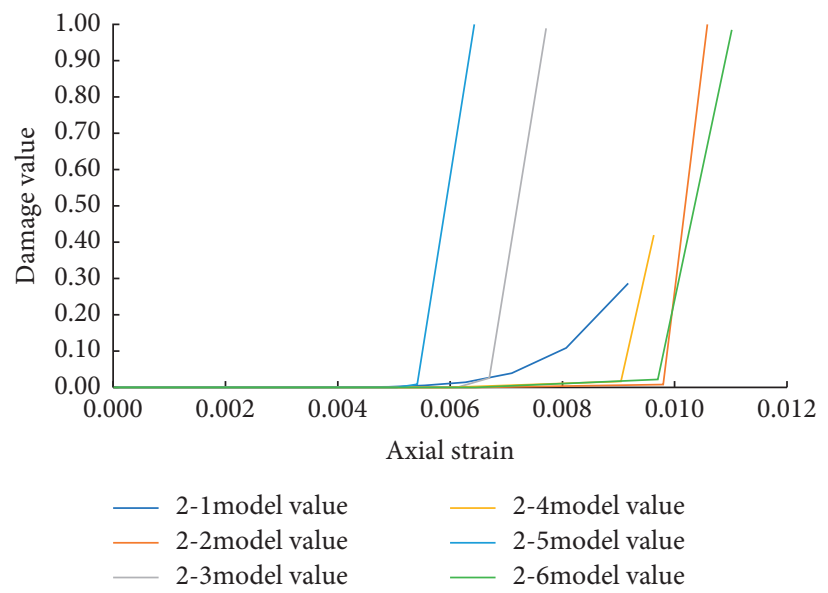

Figure 9: Theoretical curve of filled cracked rocks with cement mortar.

Comparing the curve shapes of Figures 6 and 8, after the plastic phase begins, the slope values of the test damage curve and the theoretical damage curve are the same. The same phenomenon also occurs in Figures 7 and 9. It shows that the value of the elastic modulus $E_{V}$ of the model in the plastic phase is appropriate.

The results show that (1) since the Weibull statistical model considers the initial rock damage, the rock damage 
TABLe 2: The final damage value of the test curve and model curve.

\begin{tabular}{lcccccccccccc}
\hline Sample & $1-1$ & $1-2$ & $1-3$ & $1-4$ & $1-5$ & $1-6$ & $2-1$ & $2-2$ & $2-3$ & $2-4$ & $2-5$ & $2-6$ \\
\hline$D_{\text {test }}$ & 0.77 & 0.31 & 0.61 & 0.10 & 0.37 & 0.35 & 0.24 & 0.19 & 0.15 & 0.18 & 0.17 & 0.14 \\
$D_{\text {model }}$ & 0.77 & 0.34 & 0.62 & 0.08 & 0.77 & 0.45 & 0.28 & 1.00 & 0.98 & 0.42 & 1.00 & 0.98 \\
\hline
\end{tabular}

threshold characteristics are reflected in the model damage evolution curve. (2) The model curve reflects the rock damage characteristics affected by stress and the damage characteristics affected by structural plane characteristics, and the results are the same as those of the test curve. (3) From the model curve, in the elastic stage, the damage factor $D$ of the fractured rock filled with cement mortar is 0 . This is consistent with the apparent phenomenon observed, and the rock produces fewer microcracks. (4) This model is suitable for describing the damage evolution characteristics of filled fractured rock, and it can characterize the change law of the damage with the confining pressure, the filling, and the structural surface. According to the final damage value of the curve (as shown in Table 2), it is found that the established model can more accurately characterize the fractured rock with weak and soluble fillings. However, the calculated final damage value is relatively high and inaccurate for the fractured rock filled with hydraulic materials.

\section{Discussion}

According to the Weibull distribution of damage variables and the seepage failure characteristics of the filled fractured rock, a new statistical damage model is established by using equivalent model to calculate the elastic modulus parameters. Comparing the model calculation results with the experimental data, it is found that (1) because different elastic modulus parameters are used in different stages, the model stress-strain curve fits well with the test stress-strain curve. Then, the model curve is generally in good agreement with the test curve of the fractured rock filled with gypsum mortar. The model curve fits a bit poorly with the test curve of the one filled with cement mortar, and the calculated final damage is much higher than the test data value. (2) In literature [30], the statistical model established by lognormal distribution is used to calculate the stress-strain curve of fractured rock with hydraulic material fillings, which is similar in shape to the test curve, and the final damage value is also consistent. However, the lognormal model used to calculate the fractured rock filled with gypsum mortar has a poor fit. It shows that the Weibull function is more suitable for the strength distribution characteristics of fractured rock with weak and soluble fillings. However, the lognormal function is more consistent with the strength distribution characteristics of fractured rock with hydraulic material fillings.

\section{Conclusions}

Based on the Weibull distribution of rock, the microunit strength, this paper establishes a mechanical analysis model of damage coupling. By comparing and analyzing the triaxial seepage experiment data and model calculation data of filled fractured rock, such as stress-strain curve, damage evolution curve, and final damage value, the following conclusions can be drawn:

(1) In the elastic phase, the $E_{V R H}$ parameter model can fit the test data well. In the plastic stage, the $E_{V}$ parameter model is more suitable to characterize the strength characteristics of fractured rock with low confining pressure and weak fillings.

(2) This new damage mechanics model that obeys the Weibull statistical distribution is more suitable for characterizing fractured rocks with soluble fillings.

(3) The statistical model using equivalent elastic modulus parameters can not only reflect the characteristics of the rock damage degree affected by the stress state and the type of structural surface but also reflect the characteristics of the rock damage threshold. The model can accurately calculate the final damage value of the fractured rock with weak and soluble fillings, but the final damage value of the fractured rock with hydraulic material fillings is too high and inaccurate.

\section{Data Availability}

The demand data used to support the findings of this study are available from the first author upon request.

\section{Conflicts of Interest}

The authors declare that there are no conflicts of interest regarding the publication of this paper.

\section{Acknowledgments}

This study was supported by Scientific Research Foundation of Zhejiang University of Water Resources and Electric Power, the National Natural Science Funds (51878249) of China, the Natural Science Foundation of Jiangsu Province (BK20161508), and the Postgraduate Research and Practice Innovation Program of Jiangsu Province (2018B661X14).

\section{References}

[1] J. W. Dougill, J. C. Lau, and N. J. Burt, "Toward a theoretical model for progressive failure and softening in rock, concrete and similar material," Mechanics in Engineering, vol. 102, 1976.

[2] J. Mazars, D. J. Boerman, and G. Piatti, Mechanical Damage and Fracture of concrete Structures, ICF5 Cannes (France), Cannes, France, 1981. 
[3] K. E. Loland, "Continuous damage model for load-response estimation of concrete," Cement and Concrete Research, vol. 10, no. 3, pp. 395-402, 1980.

[4] H. D. Bui, A. Ehrlacher, and C. Renard, "The steady state propagation of a damaged zone of an elastic brittle solid," Fracture 84, vol. 2, pp. 1061-1067, 1984.

[5] G. Frantziskonis and C. S. Desai, "Constitutive model with strain softening," International Journal of Solids and Structures, vol. 23, no. 6, pp. 733-750, 1987.

[6] D. Krajcinovic and M. A. G. Silva, "Statistical aspects of the continuous damage theory," International Journal of Solids and Structures, vol. 18, no. 7, pp. 551-562, 1982.

[7] W Xu and W. E. I. Lide, "Study on statistical damage constitutive model of rock," Chinese Journal of Rock Mechanics and Engineering, vol. 21, no. 6, pp. 787-791, 2002.

[8] C. A. O. G. Wen, M. H. Zhao, and L. I. U. X. Cheng, "Modified Drucker-Prager strength criterion based on statistical damage theory for rock," Journal of Hydraulic Engineering, vol. 9, no. 3, pp. 18-23, 2004.

[9] C. A. O. G. Wen, S. Zhang, and M. H. Zhao, "Study on statistical damage constitutive model of rock based on new definition of damage," Rock and Soil Mechanics, vol. 27, no. 1, pp. 41-46, 2006.

[10] C. A. O. G. Wen, S. Zhang, and M. H. Zhao, "Study on a statistical damage constitutive model with conversion between softening and hardening properties of rock," Engineering Mechanics, vol. 23, no. 11, pp. 110-115, 2006.

[11] C. G. Wen, H. Zhao, Y. J. Zhang, and L. Zhang, "Strain softening and hardening damage constitutive model for rock considering effect of volume change and its parameters determination method," Rock and Soil Mechanics, vol. 32, no. 3, pp. 647-654, 2011.

[12] W. Cao, H. Zhao, L. Xiang, and L. Zhang, "A statistical damage simulation method for rock full deformation process with consideration of the deformation characteristics of residual strength phase," China Civil Engineering Journal, vol. 45, no. 6, pp. 139-145, 2012.

[13] Z. L. Wang, Y. C. Li, and J. G. Wang, "A damage-softening statistical constitutive model considering rock residual strength," Computers \& Geosciences, vol. 33, no. 1, pp. 1-9, 2007.

[14] S. Chen, C. Qiao, Q. Ye, and M. U. Khan, "Comparative study on three-dimensional statistical damage constitutive modified model of rock based on power function and Weibull distribution," Environmental Earth Sciences, vol. 77, no. 3, 2018.

[15] J. P. Zuo, X. S. Wang, D. Q. Mao, C. L. Wang, and G. H. Jiang, "T-M coupled effects on cracking behaviors and reliability analysis of double-notched crustal rocks," Engineering Fracture Mechanics, vol. 158, no. 6, pp. 106-115, 2016.

[16] C. B. Li, L. Z. Xie, L. Ren, and J. Wang, "Progressive failure constitutive model for softening behavior of rocks based on maximum entropy theory," Environmental Earth Sciences, vol. 73, no. 10, pp. 5905-5915, 2015.

[17] Q. Sun and S. Zhu, "Wave velocity and stress/strain in rock brittle failure," Environmental Earth Sciences, vol. 72, no. 3, pp. 861-866, 2014.

[18] T. Xu, P. G. Ranjith, P. L. P. Wasantha, J. Zhao, C. A. Tang, and W. C. Zhu, "Influence of the geometry of partiallyspanning joints on mechanical properties of rock in uniaxial compression," Engineering Geology, vol. 167, pp. 134-147, 2013.

[19] K. Wu, Z. Shao, S. Qin et al., "An improved non-linear creep model for rock applied to tunnel displacement prediction,"
International Journal of Applied Mechanics, vol. 13, Article ID 2150094, 2021.

[20] Z. Chu, Z. Wu, Q. Liu, L. Weng, Z. Wang, and Y. Zhou, "Evaluating the microstructure evolution behaviors of saturated sandstone using NMR testing under uniaxial short-term and creep compression," Rock Mechanics and Rock Engineering, vol. 54, no. 9, pp. 4905-4927, 2021.

[21] A. J. Cross, S. Ellis, and D. J. Prior, "Damage and plastic deformation of reservoir rocks: Part 1. Damage fracturing," AAPG Bulletin, vol. 96, no. 9, pp. 1687-1709, 2012.

[22] Y. Wang, Y. F. Yi, C. H. Li, and J. Q. Han, "Anisotropic fracture and energy characteristics of a Tibet marble exposed to multi-level constant-amplitude (MLCA) cyclic loads: a labscale testing," Engineering Fracture Mechanics, vol. 244, no. 10, Article ID 107550, 2021.

[23] X. Fan, Z. Yang, and K. Li, "Effects of the lining structure on mechanical and fracturing behaviors of four-arc shaped tunnels in a jointed rock mass under uniaxial compression," Theoretical and Applied Fracture Mechanics, vol. 112, Article ID 102887, 2021.

[24] X. Fan, X. Jiang, Y. Liu, H. Lin, K. Li, and Z. He, "Local stress distribution and evolution surrounding flaw and opening within rock block under uniaxial compression," Theoretical and Applied Fracture Mechanics, vol. 112, Article ID 102914, 2021.

[25] K. Du, C. Yang, R. Su, M. Tao, and S. Wang, "Failure properties of cubic granite, marble, and sandstone specimens under true triaxial stress," International Journal of Rock Mechanics and Mining Sciences, vol. 130, Article ID 104309, 2020.

[26] K. Du, X. Li, M. Tao, and S. Wang, "Experimental study on acoustic emission (AE) characteristics and crack classification during rock fracture in several basic lab tests," International Journal of Rock Mechanics and Mining Sciences, vol. 133, Article ID 104411, 2020.

[27] N. Wu and J. Fu, "Research on the impact response and chaos characteristics in the dynamic damage evolution process of concrete material," Construction and Building Materials, vol. 262, no. 11, Article ID 120921, 2020.

[28] X. Liu, Z. Zhu, and A. Liu, "Permeability characteristic and failure behavior of filled cracked rock in the triaxial seepage experiment," Advances in Civil Engineering, vol. 2019, Article ID 3591629, 12 pages, 2019.

[29] Y. Chen, T. Huang, and E. Liu, Rock Physics, Press of USTC, Anhui, China, 2009.

[30] X. Liu, Z. Zhu, A. Liu, and Y. Tian, "Lognormal distribution function for describing seepage damage process of singlecracked rock," Advances in Civil Engineering, vol. 2020, Article ID 8838670, 11 pages, 2020. 